\title{
Körperliche Bestrafungen in der Verhaltenstherapie?
}

\author{
Zu Heinrichs N: Ein Klaps kann eine angemessene Disziplinierungsmaßnahme sein. \\ Ein Interview mit Robert Larzelere. Verhaltenstherapie 2010;20:61-64
}

\author{
Hans Watzl Rudolf Cohen \\ FB Psychologie, Universität Konstanz, Deutschland
}

Als wir den Titel des Interviews, «Ein Klaps kann eine angemessene Disziplinierungsmaßnahme sein», lasen, dachten wir, die Zeitschrift Verhaltenstherapie habe wieder einmal ein höchst aktuelles Thema aufgegriffen. In den letzten Monaten hatten doch die deutschen Massenmedien in immer kürzeren Abständen zunächst über sexuelle, dann auch über körperliche Misshandlungen in deutschen Internaten und Heimen berichtet. Wurde mit dem Titel die Stellungnahme eines kirchlichen Würdenträgers aufgegriffen? Das Editorial von Heinrichs und Ehlert sowie mehrere Beiträge des Heftes ließen durchaus eine wissenschaftlich geprägte Auseinandersetzung mit diesem aktuellen Thema erwarten. Dieses Interview, um das es hier geht, wird aber seltsamerweise im Editorial nicht erwähnt.

Was hat uns an diesem Interview so irritiert, dass wir in diesem Leserbrief dazu Stellung beziehen wollen? Larzelere unterscheidet zunächst zwischen Schlagen oder Hauen («spanking») einerseits und «körperlicher Bestrafung, die physisch nicht verletzend ist, eine Verhaltensänderung anstrebt und mit der flachen Hand auf die Extremitäten oder den Hintern ausgeführt wird» (p 61) andererseits. Ähnliche Formulierungen wurden in den Erklärungen von Heimleitern, Chorleitern, Priestern und Betreuungspersonal benutzt, die zuletzt in der Presse zu lesen waren.

Erstaunlicherweise wird im ganzen Interview so getan, als sei die Anwendung von körperlicher Bestrafung nur eine Frage der «Effektivität», und nicht ganz entscheidend auch eine Frage der Moral. Diese Auffassung macht uns fassungslos. Es ist zu hoffen, dass keine Zeitschrift ein Manuskript publizieren würde, das mit objektiven Daten belegt, dass die Leistung von Frauen in Betrieben davon unbeeinflusst ist, ob man sie nun 5, 10 oder 20\% schlechter bezahlt als männliche Angestellte. Dürfte man daraus wissenschaftliche Empfehlun- gen für angemessene Entlohnungen ableiten? Die beiden kriminologischen Beiträge im gleichen Heft zeigen deutlich, dass sich hinsichtlich der moralischen Einschätzung von körperlicher Bestrafung in den letzten Jahren eine ganze Menge geändert hat - was die heutige Empörung über meist länger zurückliegende Erziehungspraktiken in Heimen und Internaten mit erklärt. Diese mittlerweile bestehende Sensibilisierung gegenüber körperlicher Bestrafung darf man bei diesem Thema nicht ausblenden.

Es ist klar, dass in einem Interview Probleme nicht so eingehend und differenziert abgehandelt werden können wie in einem Sammelreferat. Dennoch stellen die meisten Aussagen von Larzelere in diesem Interview nach unserer Einschätzung so unkritische Generalisierungen dar, dass sie in dieser Form nicht erhellend, sondern nur ärgerlich wirken. So auf Seite 64: «Ältere Literaturübersichten haben gezeigt, dass Strafen grundsätzlich wirksam sind und dass die negativen Nebenwirkungen leicht minimiert werden können.» Dieser Satz kann genauso gut oder schlecht bei fast allen kritisch diskutierten Maßnahmen zur Beeinflussung von Verhalten und Erleben eingesetzt werden (Neuroleptika, Elektrokrampf-Behandlung etc.), da er letztlich nichts aussagt, was interessieren sollte. Wer würde eine solche Aussage über irgendeine pharmakologische oder sonstige medizinische Behandlung wagen? Selbst in der Werbung ist es inakzeptabel, Nebenwirkungen herunterzuspielen. Solche Aussagen durchziehen das ganze Interview: «... waren die Auswirkungen von nichtphysischen Strafen, Psychotherapie, Ritalin und Schlagen auf die Kinder ähnlich» (p 62); «Stattdessen haben Psychologen den Klaps als Möglichkeit, eine Disziplinierungsmaßnahme durchzusetzen, aufgrund von unangemessenen korrelativen Befunden fallengelassen» (p 63). Es fragt sich, wie solche Stellungnahmen unkommentiert in eine therapeutisch ausgerichtete und wissen-

\section{KARGER}

Fax +497614520714

Information@Karger.de

www.karger.com (c) 2010 S. Karger GmbH, Freiburg

Accessible online at:

www.karger.com/ver
Dr. Hans Watz

Universität Konstanz

FB Psychologie, Fach D27

78457 Konstanz, Deutschland

Tel. +49 7531 88-4620

hans.watzl@uni-konstanz.de 
schaftlich fundierte deutsche Fachzeitschrift geraten, und es ist zu hoffen, dass Sätze, wie die von uns zitierten, nicht von Massenmedien aufgegriffen werden.

Nun könnte man sagen, dass die ganzen Fragen körperlicher Strafen mit Verhaltenstherapie wenig zu tun haben, eher in den Bereich der Pädagogischen Psychologie gehören und dort diskutiert werden sollten. Diese Sicht macht das Interview keinesfalls besser, aber man müsste sich dann nicht mit den alten Vorurteilen über die mangelnde Humanität der technologieverpflichteten Verhaltenstherapie herumschlagen. Aversionsbehandlungen und ähnliche Interventionen waren hierzulande erfreulicherweise niemals von praktischer Bedeutung, sie haben aber in den Lehrbüchern der 1960er und 1970er Jahre einen breiten Raum eingenommen. Tatsächlich kommt kein Standardwerk der Pionierzeit der Verhaltenstherapie ohne entsprechende Kapitel aus: Eysenck und Rachman [1971] - «Vermeidungslernen und Aversionsbehandlung» (pp 143-154), Bandura [1969] - «Aversive Control» (pp 293354) und «Aversive Counterconditioning» (pp 501-563), Kanfer und Phillips [1970] - «Behavior Control by Aversive Consequences», Kraiker [1974] - «Bestrafung» (pp 33-84). Es gab damals kaum ein Problemverhalten, bei dem nicht Aversionsbehandlungen als Möglichkeit diskutiert wurden (z.B. bei Schreibkrampf, Stimmenhören, Homosexualität und Pädophilie, Alkoholmissbrauch, Autismus). Mitunter verschwammen damals auch die Grenzen zwischen zweifelhafter Sonderpädagogik (Lovaas' Autismus-Therapie) und Verhaltenstherapie. Damit wurden Vorurteile in der Öffentlichkeit und von anderen psychotherapeutischen Richtungen gefördert, dass ethische Erwägungen in der Verhaltenstherapie hintangestellt würden.

Heutzutage wirken fast alle dieser Interventionen grotesk. Sie ignorieren oft den gesunden Menschenverstand, widersprechen lerntheoretischen Befunden zu Diskriminationslernen und Generalisierung, und sie berücksichtigen die Bedeutung von Attributionsprozessen nicht. Die Verbreitung der Anwendung «aversiver Interventionen» war aber tatsächlich stets sehr gering. Aversionstherapien werden nach unse- rer Kenntnis in Deutschland seit Jahrzehnten nirgendwo angewandt und wurden wahrscheinlich nie außerhalb von Forschungseinrichtungen eingesetzt. Die Diskussion über den Einsatz aversiver Verfahren war deshalb zu Recht eingeschlafen, was dem Ansehen unserer Berufsgruppe gut getan hat. Larzelere wirft diese Diskussion neu auf. Obwohl er sich offenbar weitgehend auf Studien über elterliches «Erziehungsverhalten» bezieht, bringt er immer wieder die Verhaltenstherapie mit ins Spiel: «vier Therapiestudien ..., die dafür sprechen, eine gewisse Form von Schlagen anzuwenden» (p 62); «Verhaltenstherapeutische Elternschulungen haben das gleiche Ziel ...» (p 63).

Es gibt nach heutiger, gut fundierter Auffassung sicher kein Therapieziel bei irgendeinem Problemverhalten, das ausschließlich mit «Aversionsbehandlung» zu erreichen wäre. Die Darstellung von körperlichen Strafen als akzeptables, weil wirksames Mittel der Verhaltenskontrolle ignoriert somit nicht nur das damit untrennbar verbundene moralische Dilemma; ignoriert wird auch der in vielen Jahren erarbeitete, evidenzbasierte Fundus von Methoden der Verhaltensbeeinflussung, die gänzlich ohne den Einsatz aversiver Konsequenzen auskommen. Schließlich werden in dem Interview deutsche Rechtsnormen in Frage gestellt: Seit dem Jahr 2000 gesteht das BGB in $\S 1631$ allen Kindern ein «Recht auf gewaltfreie Erziehung» zu. Es ist daher im Interesse aller hierzulande tätigen Verhaltenstherapeuten, sich von den Positionen Larzeleres in diesem missverständlichen und peinlichen Interview zu distanzieren.

\section{Literatur}

Bandura A: Principles of Behavior Modification. London, Holt, Rinehart and Winston, 1969.

Eysenck HJ, Rachman S: Neurosen - Ursachen und Heilmethoden. Berlin, VEB Deutscher Verlag der Wissenschaften, 1971.

Kanfer FH, Phillips JS: Learning Foundations of Behavior Theory. New York, Wiley, 1970.

Kraiker C: Handbuch der Verhaltenstherapie. München, Kindler, 1974. 